\title{
FELICIDAD SUBJETIVA DESPUÉS DE VIVIR UN EVENTO TRAUMÁTICO EN PERSONAS CON Y SIN ANIMALES DE COMPAÑÍA
}

\section{SUBJECTIVE HAPPINESS AFTER LIVING A TRAUMATIC EVENT IN PEOPLE WITH AND WITHOUT COMPANION ANIMALS}

\author{
MÓNICA TERESA GONZÁLEZ-RAMÍREZ ${ }^{1}$, \\ LUCÍA DEL CARMEN QUEZADA-BERUMEN ${ }^{1} \mathrm{Y}$ \\ RENÉ LANDERO-HERNÁNDEZ ${ }^{1}$
}

Cómo referenciar este artículo/How to reference this article:

González-Ramírez, M. T., Quezada-Berumen, L. C. y Landero-Hernández, R. (2019). Felicidad subjetiva después de vivir un evento traumático en personas con y sin animales de compañía [Subjective Happiness after Living a Traumatic Event in People with and without Companion Animals]. Acción Psicológica, 16(1), 91-104. https://doi.org/10.5944/ap.16.1.23440

\begin{abstract}
Resumen
La interacción con los animales de compañía ha resultado efectiva en la reducción de síntomas estrés postraumático, e depresión y ansiedad, consecuentes a la vivencia de un evento traumático; aunque las investigaciones sobre aspectos positivos diferenciales entre personas con y sin animales de compañía han mostrado resultados heterogéneos. Es así que el objetivo del estudio fue comparar los niveles de felicidad subjetiva entre personas
\end{abstract}

con y sin animales de compañía, quienes habían enfrentado un suceso traumático en algún momento de los últimos tres años. Participaron 98 personas residentes en México, 49 tenían perro o gato cuando sucedió el evento y 49 no tenían. Su edad promedio fue 31.4 años $(D . E .=9.3), 65.3 \%$ mujeres y $34.7 \%$ hombres. El principal resultado indica que quienes tenían animales de compañía al momento del evento se consideran actualmente más felices que quienes no tenían. Asimismo, se encontró una correlación positiva y significativa entre felicidad y la percepción de la gente sobre la relación con el animal de compañía. Se concluye que los animales de

Correspondence address [Dirección para correspondencia]: Mónica Teresa González Ramírez. Universidad Autónoma de Nuevo León, México. Email: monygzz77@yahoo.com

ORCID: Mónica Teresa González-Ramírez (http://orcid.org/0000-0002-9058-9626), Lucía del Carmen QuezadaBerumen (http://orcid.org/0000-0003-4705-3225) y René Landero-Hernández (http://orcid.org/0000-0002-9694-8116).

${ }^{1}$ Universidad Autónoma de Nuevo León, México.

Recibido: 11 de junio de 2018.

Aceptado: 17 de octubre de 2018. 
compañía pueden ayudar a la gente a lograr un crecimiento personal posterior a un evento traumático o estresante.

Palabras clave: Eventos traumáticos; Estrés postraumático; Felicidad; Vínculo humano-animal.

\begin{abstract}
The interaction with companion animals has been effective in reducing symptoms of depression, anxiety and post-traumatic stress disorder resulting from the experience of a traumatic event; although research about differences in positive aspects between pet owners and non-pet owners have shown heterogeneous results. Thus, the objective of the study was to compare subjective happiness perceived between pet owners and non-owners, who had lived a traumatic event at some point in the last three years. Ninety-eight people residing in Mexico, recruited through a non-probabilistic sampling, participated. Half of them had dogs or cats when the event happened and 49 did not have it. Age mean was 31.4 years $(S D=9.3), 65.3 \%$ women and $34.7 \%$ men. The main result indicates that those who had companion animals at the time of the event are currently considered themselves happier than those who did not. Likewise, a positive and significant correlation was found between happiness and people's perception of the relationship with the companion animal. It is concluded that companion animals could help people achieve personal growth after a traumatic or stressful event.
\end{abstract}

Keywords: Traumatic event; Post-traumatic stress; Happiness; Human-animal bond.

\section{Introducción}

Experimentar un suceso traumático puede generar una serie de consecuencias psicológicas adversas, como la depresión, ansiedad y el trastorno de estrés postraumático (TEPT) (Ehntholt y Yule, 2006). La literatura ha mostrado que la interacción con un animal de compañía ha resultado efectiva en la reducción de dichos síntomas, así como de otros problemas de salud (Allen, 2003; Friedmann y Son, 2009; Leftowitz, Paharaia, Prout, Debiak y Bleiberg, 2005; Parshall, 2003; Souter y Miller, 2007).

Algunos investigadores han propuesto que el tener un animal de compañía está asociado con niveles más bajos de depresión (Clark Cline, 2010), mientras que otros no han encontrado una relación (Siegel, Angulo, Detels, Wesch y Mullen, 1999). Por su parte, Hart (2010) informó que quienes viven con gatos reportaron menos sentimientos de soledad, ansiedad y depresión a diferencia de aquellos que no tienen gatos.

Las personas con TEPT suelen tener condiciones de salud mental comórbidas, como trastornos del estado de ánimo, ansiedad y los trastornos por consumo de nicotina, drogas y alcohol (Smith, Goldstein y Grant, 2016) donde las consecuencias pueden ser disminución del funcionamiento de la salud mental, mala calidad de vida e incapacidad para reintegrarse plenamente en la sociedad (Gellis, Mavandadi y Oslin, 2010; Goldberg et al., 2016).

Entre las poblaciones con más evidencia científica sobre el TEPT se encuentran los veteranos de guerra, los estudios con esta población son de relevancia por su incidencia de TEPT, así como sus implicaciones. En un grupo de 30 veteranos se encontró que éstos informaron sentirse más tranquilos, menos solos, deprimidos y menos preocupados por su seguridad y la de su familia desde que su compañero perro llegó a vivir con ellos (Stern et al., 2013). Otros estudios muestran que militares activos y veteranos con perro de servicio, tenían una sintomatología de TEPT significativamente menor que aquellos sin perro de servicio, además de un mayor bienestar psicosocial, menos sintomatología depresiva, mayor funcionamiento social y una mejor calidad de vida en general (O'Haire y Rodriguez, 2018; Rodriguez, Bryce, Granger y O'Haire, 2018).

En este sentido, se ha propuesto que los animales también tienen el potencial de mejorar el bienestar general y la conexión social. En términos de bienestar psicológico, Epley, Akalis, Waytz y Cacioppo (2008) encontraron que cuando las personas eran inducidas experimentalmente a sentirse solas, eran más propensas a ver a sus animales de compañía como una fuente de apoyo. Por su parte, 
McConnell, Brown, Shoda, Stayton y Martin (2011), encontraron que los animales de compañía disminuían en sus dueños la negatividad causada por el rechazo social. En este sentido, Kanat-Maymon, Antebi y Zilcha-Mano (2016) encontraron que cuando las personas que tenían animales de compañía experimentaban una mayor necesidad de apoyo por parte de su éste, tenían un mayor bienestar. Sin embargo, el apoyo de los animales de compañía no se asoció a una menor angustia psicológica en sus dueños.

La literatura sobre los aspectos positivos del bienestar mental, como el bienestar subjetivo o la felicidad es poca. Los estudios que han evaluado la felicidad a menudo han encontrado hallazgos mixtos (McConnell et al., 2011). En una muestra representativa a nivel nacional, el Pew Research Center no encontró diferencias entre quienes tienen animales de compañía y quienes no los tienen, respecto al porcentaje de personas que se identificaron como "muy felices" (Pew Research Center, 2006). De igual forma Bao y Schreer (2016) no encontraron diferencias significativas en felicidad, emociones positivas o emociones negativas entre quienes tienen animales de compañía y quienes no los tienen. En los dos estudios anteriores, tampoco encontraron diferencias en la felicidad entre los dueños de gatos y perros (Bao y Schreer, 2016; Pew Research Center, 2006).

Los humanos forman fuertes vínculos con los animales. Esta compleja interacción se ha evidenciado durante desastres naturales, donde las personas arriesgan sus propias vidas para salvar a las de los animales o pierden la vida intentándolo (Heath, Voeks y Glickman, 2001; Heath, Kass, Beck y Glickman, 2001).

Varios estudios reportan los efectos que tienen los animales sobre algunos trastornos psiquiátricos en las personas. Sin embargo, la evidencia sobre el impacto que pueden tener los animales de compañía sobre el bienestar y la felicidad es heterogénea. Por lo anterior, realizar estudios donde se indague este efecto positivo, aportaría información que contribuya a un mejor entendimiento de la compleja interacción entre las personas y sus animales de compañía.

La investigación sobre el vínculo humano-animal puede traer beneficios. Así, el desarrollar programas de apoyo oportunos y adecuados para incentivar una mejor recuperación después de alguna situación traumática, así como promover la resiliencia), son aspectos clave para mejorar la calidad de vida de las personas. Por lo antes mencionado, el objetivo del presente estudio consistió en comparar los niveles de felicidad subjetiva entre personas que tenían animales de compañía y quienes no los tenían, después de experimentar un suceso traumático.

\section{Método}

\section{Participantes}

Se utilizó un muestreo no probabilístico completándose una muestra intencional de 98 personas residentes en México. Los criterios de inclusión fueron: ser mayor de edad (18 años) y menor de 50 años, haber vivido o ser testigo de un evento considerado como traumático en los últimos tres años y valorar su gravedad como moderada, fuerte o extrema. Se excluyó a quienes lo valoraron como leve.

Se incluyeron en el estudio 98 personas, de los cuales 49 tenían mascota cuando sucedió el evento traumático y 49 no tenían. La edad promedio de los participantes fue 31.4 años $(D . E .=9.3), 65.3 \%$ mujeres y $34.7 \%$ hombres. En cuanto a su estado civil, $49.0 \%$ eran solteros, $44.9 \%$ casados o en unión libre, $3.1 \%$ viudos y $3.0 \%$ separados o divorciados al momento de la evaluación.

\section{Instrumentos}

Para identificar el tipo de evento traumático experimentado por las personas, se utilizó la Lista de Verificación de Eventos de vida para DSM-5 (LEC-5) de Weathers et al. (2013). La lista consta de 16 situaciones estresantes que pueden suceder a lo largo de la vida, así la opción de incluir otra situación no enlistada. La persona debe indicar si la situación le sucedió a ella o fue testigo. Adicionalmente la persona puede indicar si aprendió algo del evento, si fue parte de su trabajo, si no está seguro de haberlo experimentado o si no le ha sucedido. Para este estudio, se realizó la traducción al español de la LEC-5 mediante el método de traducción inversa. 
Para evaluar felicidad se utilizó la Escala de Felicidad Subjetiva (SHS; Lyubomirsky y Lepper, 1999), en su versión validada para México (Quezada, Landero-Hernández y González-Ramírez, 2016). La escala mide la felicidad subjetiva global por medio de declaraciones, con las que los participantes se autocalifican o se comparan con los demás. Se compone de 4 ítems tipo Likert, con un coeficiente alfa de .77 (Quezada et al., 2016).

Se aplicó la escala Monash de relación del dueño con su perro (The Monash dog owner relationship scale MDORS-; Dwyer, Bennett y Coleman, 2006), en su versión para México (MDORS-M; González-Ramírez, Vanegas-Farfano y Landero-Hernández, 2017), que ha sido considerado el instrumento más robusto para la evaluación de la relación humano-perro, desde la percepción del humano sobre la relación (Payne, Bennett y McGreevy, 2015). La escala se basa en la Teoría del Intercambio Social, que establece que las relaciones humanas se mantienen sólo cuando el costo percibido y los beneficios obtenidos están balanceados o cuando los beneficios sobrepasan los costos percibidos. Consiste en una escala de 28 ítems tipo Likert, con opciones de respuesta que van de 1 a 5, indicando el grado de acuerdo o bien que tan frecuente el dueño interactúa con el perro. Se compone de tres subescalas: (1) interacción dueño-perro; (2) cercanía emocional percibida; (3) Costo percibido. Sin embargo, para los propósitos del estudio solo se aplicaron las primeras dos subescalas. Para la versión Mexicana, el alfa de Cronbach fue de .82 en la subescala de interacción dueñoperro; de 91 para la cercanía emocional (González-Ramírez et al., 2017).

Se incluyeron preguntas sobre la percepción de la severidad del evento (de acuerdo a tu percepción, ¿qué tan severo o grave fue el evento ocurrido?), el tiempo transcurrido desde el evento (¿cuánto tiempo ha transcurrido desde el suceso traumático?), si habían recibido acompañamiento psicológico de algún profesional (desde que sucedió el evento ¿has recibido acompañamiento psicológico de algún profesional?), el tipo de animal de compañía que tenían (¿qué tipo de mascota tenías?) y dos preguntas sobre la relación con el animal de compañía, (del 0 al 10 ¿qué tanto consideras que la relación con tu mascota representó un apoyo para afrontar situaciones estresantes? y del 0 al 10 ¿qué tan buena considerabas la relación con esa mascota?)

\section{Procedimiento}

Se invitó a responder una encuesta en línea a personas adultas residentes en México. La encuesta fue realizada a través de SurveyMonkey.com y difundida a través de redes sociales, siguiéndose un método de muestreo guiado por el participante, donde a cada persona se le pedía invitar a otra a participar. Se descartaron las encuestas que no llegaron a la última pregunta o que no cumplieran los criterios de inclusión mencionados. Todos los datos se trataron siguiendo los principios éticos de la Asociación Americana de Psicología (2017).

\section{Análisis estadístico}

Se realizaron pruebas no paramétricas debido a que las variables analizadas no se ajustaron a una distribución normal de acuerdo a los resultados de la prueba de Kolmogorov-Smirnov $(p<.01)$. Además de analizar las diferencias en felicidad subjetiva entre quienes tenían y quienes no tenían animales de compañía, se analizaron algunas posibles variables extrañas que pudieran impactar en el nivel reportado de felicidad. Así, para determinar la asociación entre las variables continuas, se utilizó la correlación de Spearman. Se utilizó la prueba Kruskal-Wallis para la comparación entre quienes consideraron el evento como moderado, severo o grave. Para evaluar las diferencias entre las submuestras, se utilizó la prueba Chi-cuadrada $(\chi 2)$ para variables categóricas y la prueba U de Mann-Whitney para las variables continuas, además se calculó el tamaño del efecto, utilizando la d de Cohen. Los cálculos estadísticos se realizaron con SPSS 24, con un nivel de significación o probabilidad exacta en los contrastes de .05 e intervalos de confianza para las estimaciones del $95 \%$.

\section{Resultados}

Para evaluar variables que podrían impactar en el puntaje de felicidad subjetiva, antes de analizar las diferencias 
Tabla 1

Clasificación del evento traumático de acuerdo a los grupos

\begin{tabular}{|c|c|c|c|c|}
\hline & \multicolumn{2}{|c|}{$\begin{array}{c}\text { Sin animal de } \\
\text { compañía } \\
\text { durante el evento }\end{array}$} & \multicolumn{2}{|c|}{$\begin{array}{c}\text { Con animal de } \\
\text { compañía } \\
\text { durante el evento }\end{array}$} \\
\hline & $\mathbf{n}$ & $\%$ & $\mathbf{n}$ & $\%$ \\
\hline $\begin{array}{l}\text { Desastres naturales (huracán, terremoto, inundación o deslizamientos de } \\
\text { tierra) }\end{array}$ & 6 & 12.2 & 12 & 24.5 \\
\hline Fuego o explosión & 1 & 2.0 & 0 & 0.0 \\
\hline $\begin{array}{l}\text { Accidente de transporte (por ejemplo, accidente de coche, accidente de } \\
\text { barco, accidente de tren, accidente de avión) }\end{array}$ & 8 & 16.3 & 3 & 6.1 \\
\hline Accidente grave en el trabajo, en casa o durante alguna actividad recreativa & 3 & 6.1 & 3 & 6.1 \\
\hline Ataque físico (ser atacado, golpeado, abofeteado, pateado, golpeado) & 2 & 4.1 & 3 & 6.1 \\
\hline $\begin{array}{l}\text { Ataque con un arma (haber recibido un disparo, apuñalado, amenazado con } \\
\text { un cuchillo, una pistola, una bomba) }\end{array}$ & 4 & 8.2 & 4 & 8.2 \\
\hline $\begin{array}{l}\text { Ataque sexual (violación, intento de violación, hecho para realizar cualquier } \\
\text { tipo de acto sexual a través de la fuerza) }\end{array}$ & 1 & 2.0 & 2 & 4.1 \\
\hline Otra experiencia sexual no deseada o incómoda & 2 & 4.1 & 0 & 0.0 \\
\hline Cautiverio (ser secuestrado, mantenido como rehén, prisionero de guerra) & 0 & 0.0 & 2 & 4.1 \\
\hline Enfermedad o lesión potencialmente mortal & 5 & 10.2 & 5 & 10.2 \\
\hline Sufrimiento humano severo (tortura física o psicológica) & 3 & 6.1 & 1 & 2.0 \\
\hline $\begin{array}{l}\text { Muerte violenta repentina (homicidio o suicidio de alguien cercano o } \\
\text { conocido) }\end{array}$ & 3 & 6.1 & 2 & 4.1 \\
\hline Muerte accidental repentina (de alguien cercano o conocido) & 11 & 22.4 & 12 & 24.5 \\
\hline
\end{tabular}

entre quienes tenían y no tenían mascota al momento del evento traumático, se realizaron dos análisis con la muestra completa. Se llevó a cabo un análisis de correlación entre la felicidad subjetiva y los años transcurridos desde el evento traumático, correlación que no resultó significativa $\left(r_{s}=.098, p=.337\right)$. Se realizaron análisis de comparación de grupos, encontrando puntajes equivalentes de fe-

Tabla 2

Comparación de la edad, tiempo transcurrido y felicidad subjetiva entre los grupos

\begin{tabular}{|c|c|c|c|c|c|c|c|c|c|}
\hline & \multicolumn{3}{|c|}{$\begin{array}{l}\text { Sin animal de compañía } \\
\text { durante el evento traumático }\end{array}$} & \multicolumn{3}{|c|}{$\begin{array}{l}\text { Con animal de compañía } \\
\text { durante el evento traumático }\end{array}$} & \multicolumn{2}{|c|}{$\begin{array}{l}\text { U de Mann } \\
\text { Whitney }\end{array}$} & \multirow{2}{*}{$\begin{array}{l}\text { d de } \\
\text { Cohen }\end{array}$} \\
\hline & Mediana & Media & D.E. & Mediana & Media & D.E. & $\mathbf{Z}$ & $p$ & \\
\hline Edad & 29.0 & 31.3 & 9.2 & 28.0 & 31.5 & 9.4 & -0.050 & .960 & -0.02 \\
\hline $\begin{array}{l}\text { Años transcurrido } \\
\text { desde el suceso } \\
\text { estresante o traumático }\end{array}$ & 0.7 & 1.1 & 1.0 & 0.7 & 1.1 & 0.9 & -0.418 & .676 & 0 \\
\hline Felicidad subjetiva & 20.0 & 19.3 & 4.9 & 22.0 & 21.6 & 4.4 & -2.356 & .018 & -0.49 \\
\hline
\end{tabular}

licidad subjetiva entre quienes consideraron el evento como moderado, fuerte y severo $(K W=3.086, p=.211)$, entre quienes tenían pareja y personas solteras $(Z=-1.529$, $p=.126)$, y entre hombres y mujeres $(Z=-0.427$, $p=.669)$. 
Tabla 3

Características del evento traumático

\begin{tabular}{|c|c|c|c|c|}
\hline & \multicolumn{2}{|c|}{$\begin{array}{c}\text { Sin animal de } \\
\text { compañía durante el } \\
\text { evento traumático }\end{array}$} & \multicolumn{2}{|c|}{$\begin{array}{c}\text { Con animal de compañía } \\
\text { durante el evento } \\
\text { traumático }\end{array}$} \\
\hline & $\mathbf{n}$ & $\%$ & $\mathbf{n}$ & $\%$ \\
\hline \multicolumn{5}{|l|}{ Cercanía del evento traumático } \\
\hline Le sucedió al participante & 28 & 57.1 & 29 & 59.2 \\
\hline El participante fue testigo y lo presenció directamente & 21 & 42.9 & 20 & 40.8 \\
\hline \multicolumn{5}{|l|}{ Gravedad del evento traumático } \\
\hline Moderado & 13 & 26.5 & 9 & 18.4 \\
\hline Fuerte & 23 & 46.9 & 23 & 46.9 \\
\hline Extremo o grave & 13 & 26.5 & 17 & 34.7 \\
\hline
\end{tabular}

Se comparó el tipo de evento traumático enfrentado por las personas que tenían y que no tenían animales de compañía en el momento del evento (Tabla 1); los desastres naturales fue el tipo de evento que reportaron más quienes tenían animales de compañía, mientras que para quienes no los tenían fueron accidentes de transporte. Los resultados de la prueba Chi cuadrada $\left(\chi^{2}=11.050\right.$, $p=.525)$ indican equivalencia entre ambos grupos en el tipo de eventos reportados

El principal resultado de la investigación indica que quienes tenían animales de compañía se consideran actualmente más felices que quienes no los tenían al momento del evento traumático $(Z=-2.356, p=.018)$, con un tamaño del efecto medio $(d=-0-49)$. Además de comparar los niveles de felicidad entre los grupos, se compararon otras variables; en la Tabla 2 puede observarse que los grupos eran equivalentes en edad y en años transcurridos desde el evento traumático. Considerando que la diferencia en niveles de felicidad podría explicarse por otras variables se compararon algunas variables entre los dos gru- pos: para sexo $\left(\chi^{2}=0.180, p=.671\right)$ y estado civil $(\chi 2=3.838, p=.573)$, la prueba Chi cuadrada no resultó significativa, y tampoco para acompañamiento psicológico $(\chi 2=0, p=1.00)$, para lo cual 11 personas de cada grupo reportaron haberlo recibido después del evento y 38 de cada grupo indicaron que no.

Se analizó si algunas características del evento traumático diferían entre los grupos, encontrando equivalencia entre los grupos en cuanto a cercanía del evento traumático $\left(\chi^{2}=0.042, p=.838\right)$ y la gravedad de este $\left(\chi^{2}=1.261, p=.532\right)($ Tabla 3$)$.

Finalmente, se analizaron algunas variables en el grupo de personas que tenían animales de compañía durante el evento traumático, en la Tabla 4 se presentan los estadísticos descriptivos para las variables de relación con el animal de compañía, puede observarse que las personas consideran que sus animales de compañía son un apoyo para afrontar situaciones estresantes y que su relación con ellos es buena. Todos contaban aun con el mismo el animal de

Tabla 4

Estadísticos descriptivos para las variables de relación con los animales de compañía

\begin{tabular}{|c|c|c|c|}
\hline & \multicolumn{3}{|c|}{$\begin{array}{l}\text { Con animal de compañía durante el } \\
\text { evento traumático }\end{array}$} \\
\hline & Mediana & Media & D.E. \\
\hline $\begin{array}{l}\text { Del } 0 \text { al } 10 \text { ¿qué tanto consideras que la relación con tu mascota } \\
\text { representa un apoyo para afrontar situaciones estresantes? }\end{array}$ & 7.0 & 7.3 & 2.5 \\
\hline Del 0 al 10 ¿qué tan buena consideras tu relación con esa mascota? & 10.0 & 9.0 & 2.1 \\
\hline Interacción humano-animal (MDORS) & 25.0 & 22.6 & 12.9 \\
\hline Cercanía emocional percibida (MDORS) & 39.0 & 32.8 & 16.3 \\
\hline
\end{tabular}


Tabla 5

Correlaciones de Spearman entre las variables de estudio en la submuestra de personas con animal de compañía durante el evento traumático

\begin{tabular}{|c|c|c|c|c|c|}
\hline & $\begin{array}{c}\text { Años } \\
\text { desde el } \\
\text { evento }\end{array}$ & $\begin{array}{c}\text { Animal de compañía } \\
\text { como apoyo para } \\
\text { afrontar situaciones } \\
\text { estresantes }\end{array}$ & $\begin{array}{c}\text { ¿Qué tan buena } \\
\text { consideras tu relación } \\
\text { con ese animal de } \\
\text { compañía? }\end{array}$ & $\begin{array}{c}\text { MDORS } \\
\text { Interacción } \\
\text { humano animal }\end{array}$ & $\begin{array}{l}\text { MDORS } \\
\text { Cercanía } \\
\text { emocional } \\
\text { percibida }\end{array}$ \\
\hline \multicolumn{6}{|l|}{ Mascota como apoyo } \\
\hline $\begin{array}{l}\text { para afrontar } \\
\text { situaciones } \\
\text { estresantes }\end{array}$ & .143 & 1 & & & \\
\hline $\begin{array}{l}\text { ¿Qué tan buena } \\
\text { consideras tu relación }\end{array}$ & & & & & \\
\hline $\begin{array}{l}\text { consideras tu relacion } \\
\text { con ese animal de } \\
\text { compañía? }\end{array}$ & -.079 & $.474^{* *}$ & 1 & & \\
\hline $\begin{array}{l}\text { MDORS Interacción } \\
\text { humano animal }\end{array}$ & .056 & .204 & $.377^{* *}$ & 1 & \\
\hline $\begin{array}{l}\text { MDORS Cercanía } \\
\text { emocional percibida }\end{array}$ & -.063 & .200 & .224 & $.727^{\star *}$ & 1 \\
\hline $\begin{array}{l}\text { Escala de felicidad } \\
\text { subjetiva }\end{array}$ & -.004 & .040 & $.311^{*}$ & .163 & -.039 \\
\hline
\end{tabular}

compañía al momento de la evaluación, en su mayoría perro $(n=41)$ y solamente ocho personas tenían gato

En la Tabla 5 se presentan las correlaciones de la submuestra de las personas que tenían animales de compañía al momento del evento traumático, se encontraron correlaciones positivas y significativas entre felicidad y la percepción de la gente sobre su relación con el animal de compañía $\left(r_{s}=.311, p=.029\right)$, y entre algunas de las variables sobre la relación humano-animal; algunas correlaciones fueron superiores que $.20 \mathrm{sin}$ embargo, debido al tamaño de muestra no fueron significativas.

\section{Discusión}

Los resultados mostraron que se consideran más felices aquellas personas que tenían animales de compañía al momento del evento traumático. Este resultado es interesante ya que en investigaciones previas, que han comparado personas que tenían y que no tenían animales de compañía, no se encontraron diferencia en sus puntajes de felicidad (Bao y Schreer, 2016; González-Ramírez y Landero-Her- nández, 2014). No se encontró correlación significativa entre la felicidad subjetiva y la percepción del participante sobre el apoyo recibido de su perro o gato para afrontar situaciones estresantes, pero si con la percepción sobre su relación con el animal de compañía. Es así que se considera que son más felices quienes tenían animales de compañía al momento del suceso y más aún quienes consideran que su relación es mejor, sin importar si perciben apoyo por parte de su perro o gato para sobrellevar la situación estresante.

La presente investigación pretende contribuir al estudio sobre la felicidad y a la línea de investigación sobre vínculo humano-animal, en el contexto de situaciones traumáticas o estresantes que pudieran dar origen a estrés postraumático o bien a crecimiento postraumático; para lo cual se incluyeron en el estudio personas que hubieran estado expuestas a este tipo de situaciones en algún momento durante los últimos tres años y que consideraran que la gravedad de la situación fue moderada, fuerte o extrema, excluyendo a quienes la catalogaban como leve; esta decisión se basa en los planteamientos de Lazarus y Folkman (1984) quienes consideran que las respuesta al estrés se debe a como la persona valora la situación y no a 
la situación por sí misma y a lo documentado por García Martínez, Jaramillo, Martínez, Valenzuela y Cova Solar (2014) respecto a que la percepción de severidad del evento se relaciona con el desarrollo del síndrome de estrés postraumático y también con el crecimiento postraumático debido a que la gravedad percibida del suceso activa mecanismos que permiten el crecimiento personal.

Es así que se consideró que la valoración que hace actualmente el participante sobre la gravedad de la situación pudiera estar asociada a la felicidad subjetiva, sin embargo, no fue significativa la diferencia en felicidad subjetiva entre quienes consideraron el evento como moderado, fuerte y severo; esto pudiera explicarse con los hallazgos de García Martínez et al. (2014) quienes encontraron que hay variables mediadoras entre la percepción de severidad y las consecuencias en crecimiento personal. Asimismo, el tiempo transcurrido desde el evento tampoco se relacionó con el puntaje de felicidad.

A pesar de las limitaciones propias del tipo de muestreo, tamaño de muestra y forma de recolección de datos, se concluye que los animales de compañía pueden ayudar a la gente a lograr un crecimiento personal posterior a un evento traumático o estresante. Considerando este factor, en los tratamientos para estrés postraumático se podría incluir actividades que mejoren la relación humano animal, en el caso de personas que cuenten con animales de compañía o intervenciones asistidas con animales para quienes no los tienen. Para futuras investigaciones se sugiere replicar la presente investigación en una muestra de personas con diagnóstico de estrés postraumático, explorando sintomatología específica tras el evento y evaluando a muestras mayores y representativas que permitieran la generalización de resultados.

\section{Referencias}

Allen, K. (2003). Are Pets a Healthy Pleasure? The Influence of Pets on Blood Pressure. Current Directions in Psychological Science, 12(6), 236239 https://doi.org/10.1046/j.0963-7214.2003.012 69.x
American Psychological Association (2017). Ethical principles of psychologists and code of conduct. With the 2016 amendment to standard 3.04. Washington, DC: Autor.

Bao, K. J. y Schreer, G. (2016). Pets and Happiness: Examining the Association between Pet Ownership and Wellbeing, Anthrozoös, 29(2), 283-296. https://doi.org/10.1080/08927936.2016.1152721

Clark Cline, K. M. (2010). Psychological Effects of Dog Ownership: Role strain, Role Enhancement, and Depression. The Journal of Social Psychology 150, 117-131. https://doi.org/10.1080/002245409033 68533

Dwyer, F., Bennett, P. C. y Coleman, G. J. (2006). Development of the Monash Dog Owner Relationship Scale (MDORS). Anthrozoös 19, 243-256. https://doi.org/10.2752/0892793067854 15592

Ehntholt, K. y Yule, W. (2006). Practitioner Review: Assessment and Treatment of Refugee Children and Adolescents who have Experienced WarRelated Trauma. Journal of Child Psychology and Psychiatry, 47(12), 1197-1210. https://doi.org/10. 1111/ j.1469-610.2006.01638.x

Epley, N., Akalis, S., Waytz, A. y Cacioppo, J. T. (2008). Creating Social Connection through Inferential Reproduction. Psychological Science, 19, 114120. https://doi.org/10.1111/j.1467-9280.2008. 02056.x

Friedmann, E. y Son, H. (2009). The Human-companion Animal Bond: How Humans Benefit. Veterinary Clinics: Small Animal Practice, 393(2), 293-326. https://doi.org/10.1016/j.cvsm.2008.10.015

García Martínez, F. E., Jaramillo, C., Martínez, A. M., Valenzuela, I. y Cova Solar, F. (2014). Respuestas psicológicas ante un desastre natural: Estrés y crecimiento postraumático [Psychological Responses to a Natural Disaster: Stress and Posttraumatic Growth]. Liberabit, 20(1), 121-130. 
Gellis, L. A., Mavandadi, S. y Oslin, D. W. (2010). Functional Quality of Life in Full versus Partial Posttraumatic Stress Disorder among Veterans Returning from Iraq and Afghanistan. Primary Care Companion to the Journal of clinical psychiatry, 12(3), PCC.09m00823. https://doi.org/ 10.4088/PCC.09m00823blu

Goldberg, J., Magruder, K. M., Forsberg, C. W., Friedman, M. J., Litz, B. T., Vaccarino, V., ... y Smith, N. L. (2016). Prevalence of Post-traumatic Stress Disorder in Aging Vietnam-era Veterans: Veterans Administration Cooperative Study 569: Course and Consequences of Post-traumatic Stress Disorder in Vietnam-era Veteran Twins. The American Journal of Geriatric Psychiatry, 24(3), 181-191. https://doi.org/10.1016/j.jagp.2015.05.00 4

González-Ramírez, M. T. y Landero-Hernández, R. (2014). Benefits of Dog Ownership: Comparative Study of Equivalent Samples. Journal of Veterinary Behavior: Clinical Applications and Research, 9(6), 311-315. https://doi.org/10.1016/ j.jveb.2014.08.002

González-Ramírez, M. T., Vanegas-Farfano, M. y Landero-Hernández, R. (2017). Versión mexicana de la escala Monash de relación del dueño con su perro (MDORS-M) [Mexican Version of the Monash Dog Owner Relationship Scale, MDORSM]. Alternativas en Psicología, 37, 107-123. Recuperado de http://www.alternativas.me/26numero-37-febrero-julio-2017/149-versionmexicana-de-la-escala-monash-de-relacion-deldueno-con-su-perro-mdors-m

Hart, L. (2010). Methods, standards, guidelines and considerations in selecting animals for AnimalAssisted Therapy. En A. Fine (Ed.), Handbook on animal-assisted therapy ( $1^{\mathrm{a}}$ ed., pp. 81-114). San Diego, CA: Academic Press.

Heath, S. E., Kass, P. H., Beck, A. M. y Glickman, L. T. (2001). Human and pet-Related Risk Factors for Household Evacuation Failure during a Natural
Disaster. American Journal of Epidemiology, 153(7), 659-665. https://doi.org/10.1093/aje/153. 7.659

Heath, S. E., Voeks, S. K. y Glickman, L. T. (2001). Epidemiologic Features of Pet Evacuation Failure in a Rapid-Onset Disaster. Journal of the American Veterinary Medical Association, 218(12), 18981904. https://doi.org/10.2460/javma.2001.218.189 8

Kanat-Maymon, Y., Antebi, A. y Zilcha-Mano, S. (2016). Basic Psychological Need Fulfillment in Human-pet Relationships and Well-being. Personality and Individual Differences, 92, 69-73. https://doi.org/10.1016/j.paid.2015.12.025

Lazarus, R. y Folkman, S. (1984). Stress, Coping, and Adaptation. New York, NY: Springer.

Leftowitz, C., Paharaia, I., Prout, M., Debiak, D. y Bleiberg, J. (2005). Animal-assisted Prolonged Exposure: A Treatment for Survivors of Sexual Assault Suffering Posttraumatic Stress Disorder. Society \& Animals, 13(4), 275-295. https://doi.org/ $10.1163 / 156853005774653654$

Lyubomirsky, S. y Lepper, H. S. (1999). A Measure of Subjective Happiness: Preliminary Reliability and Construct Validation. Social Indicators Research, 46, 137-155. https://doi.org/10.1023/A:10068241 00041

McConnell, A. R., Brown, C. M., Shoda, T. M., Stayton, L. E. y Martin, C. E. (2011). Friends with Benefits: On the Positive Consequences of Pet Ownership. Journal of Personality and Social Psychology 101, 1239-1252 https://doi.org/10.1037/a0024506

O’Haire, M. E. y Rodriguez, K. (2018). Preliminary Efficacy of Service Dogs as a Complementary Treatment for Posttraumatic Stress Disorder in Military Members and Veterans. Journal of Consulting and Clinical Psychology 86, 179-188. https://doi.org/10.1037/ccp0000267 
Parshall, D. (2003). Research and Reflection: AnimalAssisted Therapy in Mental Health Settings. Counselling and Values, 48, 47-55. https://doi.org/10.1002/j.2161-007x.2003.tb002 74.x

Payne, E., Bennett, P. C. y McGreevy, P. D. (2015). Current Perspectives on Attachment and Bonding in the Dog-human Dyad. Psychology research and behavior management, 8, 71-79 https://doi.org/10. 2147/PRBM.S74972

Pew Research Center. (2006). Are we Happy Yet? Recuperado de http://www.pewsocialtrends.org/2006/02/13/arewe-happy-yet/.

Quezada, L., Landero-Hernández, R. y GonzalezRamirez, M. T. (2016). A Validity and Reliability Study of the Subjective Happiness Scale in Mexico. The Journal of Happiness \& Well-Being, 4(1), 90100.

Rodriguez, K. E., Bryce, C. I., Granger, D. A. y O’Haire, M. E. (2018). The Effect of a Service Dog on Salivary Cortisol Awakening Response in a Military Population with Posttraumatic Stress Disorder (PTSD). Psychoneuroendocrinology, 98, 202-210. https://doi.org/10.1016/j.psyneuen.2018. 04.026

Siegel, J. M., Angulo, F. J., Detels, R., Wesch, J. y Mullen, A. (1999). AIDS diagnosis and depression in the Multicenter AIDS Cohort Study: The ameliorating impact of pet ownership. AIDS Care $11,157-169$.
Smith, S. M., Goldstein, R. B. y Grant, B. F. (2016). The association between post-traumatic stress disorder and lifetime DSM-5 psychiatric disorders among veterans: data from the National Epidemiologic Survey on Alcohol and Related Conditions-III (NESARC-III). Journal of psychiatric research, 82, 16-22. https://doi.org/10.1016/j.jpsychires. 2016.06.022

Souter, M. A. y Miller, M. D. (2007). Do animal-assisted activities effectively treat depression? A metaanalysis. Anthrozoos, 20, 167-180. https://doi.org/ $10.2752 / 175303707 \times 207954$

Stern, S. L., Donahue, D. A., Allison, S., Hatch, J. P., Lancaster, C. L., Benson, T. A., ... y Peterson, A. L. (2013). Potential Benefits of Canine Companionship for Military Veterans with Posttraumatic Stress Disorder (PTSD). Society \& Animals, 21(6), 568-581. https://doi.org/10.1163/ $15685306-12341286$

Weathers, F. W., Blake, D. D., Schnurr, P. P., Kaloupek, D. G., Marx, B. P. y Keane, T. M. (2013). The Life Events Checklist for DSM-5 (LEC-5) - Standard. [Measurement instrument]. Recuperado de http://www.ptsd.va.gov/professional/assessment/te-measures/life_events_checklist.asp 


\title{
SUBJECTIVE HAPPINESS AFTER LIVING A TRAUMATIC EVENT IN PEOPLE WITH AND WITHOUT COMPANION ANIMALS
}

\author{
MÓNICA TERESA GONZÁLEZ-RAMÍREZ ${ }^{1}$, \\ LUCÍA DEL CARMEN QUEZADA-BERUMEN ${ }^{1}$, AND \\ RENÉ LANDERO-HERNÁNDEZ ${ }^{1}$
}

\section{EXTENDED SUMMARY}

Companion animals have the potential to improve general well-being and social connection. There is little literature on the positive aspects of mental well-being, such as subjective well-being or happiness. Studies that have evaluated happiness have often found mixed findings (McConnell et al., 2011). In a nationally representative sample, the Pew Research Center did not find differences between those who have companion animals and those who do not with respect to the percentage of people who identified themselves as "very happy" (Pew Research Center, 2006). Likewise et al. (2016) found no significant differences in happiness, positive emotions or negative emotions between those who have companion animals and those who do not. In the two previous studies, researchers also did not find differences in happiness between cat and dog owners (Bao \& Schreer, 2016; Pew Research Center, 2006).

Humans form strong bonds with animals. This complex interaction has been evidenced during natural disasters when people have risked and even lost their own lives to save those of animals (Heath, Voeks, \& Glickman, 2001; Heath, Kass, Beck, \& Glickman, 2001).

Thus, the evidence on the impact that companion animals can have on well-being and happiness is heterogeneous. Therefore, conducting studies that investigate this positive effect would provide information that contributes to a better understanding of the complex interaction between people and their companion animals.
Research on the human-animal bond can bring benefits. Thus, developing timely and adequate support programs to encourage a better recovery after a traumatic situation as well as fostering resilience are key aspects to improving people's quality of life. Therefore, the objective of this study was to compare the levels of subjective happiness between people who had companion animals and those who did not after experiencing a traumatic event.

\section{Method}

\section{Participants}

Nonprobabilistic intentional sampling was used, reaching a sample of 98 people living in Mexico. The inclusion criteria were age above 17 years and under 50 years, as well as having lived or witnessed an event, considered by themselves as traumatic, in the last 3 years and assessing its severity as moderate, strong or extreme. Those who rated it as mild were excluded.

A total of 98 people were included in the study, of which 49 had at least one companion animal when the traumatic event occurred and 49 did not. The average age of participants was 31.4 years $(\mathrm{SD}=9.3)$, with $65.3 \%$ women and $34.7 \%$ men. For their marital status, $49.0 \%$ were single, $44.9 \%$ married or in a domestic partnership, $3.1 \%$ widowed and $3.0 \%$ separated or divorced at the time of the evaluation. 


\section{Instruments}

To identify the type of traumatic event experienced by people, the Live Events Checklist for DSM-5 (LEC-5), developed by Weathers et al. (2013), was used. To evaluate happiness, the Subjective Happiness Scale (SHS; Lyubomirsky \& Lepper, 1999) was used in its version validated for Mexico (Quezada et al., 2016). Also, the Monash dog owner relationship scale -MDORS- (Dwyer et al., 2006), in its version for Mexico was used (MDORS-M; González-Ramírez et al., 2017), which has been considered the most robust instrument for evaluating the human-dog relationship from the perception of humans (Payne et al., 2015). A few more questions were included about the perceived severity of the event, the time elapsed since the event, if they had received psychological support from a professional, and the type of pet they had as well as two questions about the relationship with the pet.

\section{Procedure}

Adults in Mexico were invited to respond to an online survey. The survey was conducted through SurveyMonkey.com and disseminated through social networks following a participant-guided sampling method in which each person was asked to invite another to participate. Individuals who did not reach the last question or who did not meet the inclusion criteria were excluded. All data were treated following the ethical principles of the American Psychological Association (2017).

\section{Results}

To evaluate variables that could influence the subjective happiness score, before analyzing the differences between those who had a pet and those who did not at the time of the traumatic event, two analyses were performed with the complete sample. A correlation analysis between subjective happiness and the years elapsed since the traumatic event was carried out. This correlation was not significant ( $\mathrm{rs}=.098, p=.337)$. A comparison analysis of groups was performed, demonstrating equivalent subjective happiness scores between those who considered the event as moderate, strong and severe $(\mathrm{KW}=3.086$, $p=.211$ ), between those who had a partner and single people $(Z=-1.529, p=.126)$, and between men and women $(Z=-0.427, p=.669)$.

The main result of the research indicated that those who had pets at the time of the traumatic event were considered happier than those who did not $(Z=-2.356$, $p=.018)$, with a medium effect size $(d=-0.49)$.

Analyses were conducted to determine whether some characteristics of the traumatic event differed between the groups and found equivalence between the groups in terms of proximity of the traumatic event $\left(\chi^{2}=0.042, p=.838\right)$ and its severity $\left(\chi^{2}=1.261, p=.532\right)($ Table 3$)$.

Finally, characteristics of pet-owner relationships were analyzed in the group of people who had pets during the traumatic event. In Table 4, descriptive statistics are presented for the variables. It can be seen that people considered their companion animals to be supportive in coping with stressful situations and that their relationship with them was good. All still had the same companion animal at the time of the evaluation. Most of the companion animals were dogs $(n=41)$, and only eight people had a cat.

In the subsample of people who had companion animals at the time of the traumatic event, positive and significant correlations were found between happiness and the perception of pet owners of their relationship with their pets (rs $=.311, p=.029)$ and between some of the variables on the human-animal relationship. Some correlations were higher than .20. However, due to sample size, they were not significant.

\section{Discussion}

The results showed that people who had companion animals at the time of a traumatic event considered themselves happier than those who did not. This result is interesting, because in previous researches that compared people who had or did not have companion animals, no difference was found in their happiness scores (Bao \& Schreer, 2016; González-Ramírez \& Landero-Hernández, 
2014). No significant correlation was found between subjective happiness and participants' perceptions of the support they received from their dog or cat when facing stressful situations, but there was a significant correlation with their perceived relationship with their companion animals. Thus, those people who had companion animals at the time of the traumatic event were happier and even more so considered their relationship to be better, regardless of whether they perceived support from their dog or cat to cope with the stressful situation.

The decision to include in the study people who had been exposed to this type of situation at some point during the past three years and who considered the severity of the situation to be moderate, strong or extreme and to exclude those who classified it as mild was based on the theory of Lazarus and Folkman (1984), who proposed that response to stress is related to how a given person assesses a situation and not the situation itself. This is documented by García Martínez et al. (2014) with regard to the fact that the perceived severity of an event is related to the development of posttraumatic stress syndrome and to posttraumatic growth, because the perceived severity of the event activates mechanisms that allow for personal growth.

Despite the limitations of this type of sampling, the sample size and the form of data collection, we can conclude that companion animals can help people achieve personal growth after a traumatic or stressful event (posttraumatic growth). Considering this factor, treatments for posttraumatic stress could include activities that improve the human-animal relationship in the case of people who have companion animals and include animal-assisted interventions for those who do not. For future studies, we suggest replicating this study in a sample of people diagnosed with posttraumatic stress, exploring specific symptomatology after the event and evaluating larger and representative samples that would allow for the generalization of the results. 
